\title{
Comparison of Some Static Path Planning Models Localization Performance in Obstacle-Presence Environment
}

\author{
Dogan Yildiz $^{1^{*}}$, Serap Karagol ${ }^{2}$ \\ 1*, Ondokuz Mayis University, Faculty of Engineering, Department of Electrical-Electronics Engineering, Samsun, Turkey, (ORCID: 0000-0001-9670-4173), \\ dogan.yildiz@omu.edu.tr \\ ${ }^{2}$ Ondokuz Mayis University, Faculty of Engineering, Department of Electrical-Electronics Engineering, Samsun, Turkey, (ORCID: 0000-0002-5750-1143), \\ serap.karagol@omu.edu.tr
}

(3rd International Congress on Human-Computer Interaction, Optimization and Robotic Applications June 11-13, 2021)

(DOI: $10.31590 /$ ejosat.960213)

ATIF/REFERENCE: Yildiz, D. \& Karagol, S. (2021). Comparison of Some Static Path Planning Models Localization Performance in Obstacle-Presence Environment. European Journal of Science and Technology, (26), 438-446.

\begin{abstract}
Static anchors are generally used for the localization of Unknown Nodes (UNNs) in Wireless Sensor Networks (WSNs). However, it would be a more efficient approach to design a Mobile Anchor (MA) trajectory to cover all UNNs instead and to have the MA travel to broadcast its position at specific points along that trajectory. With this logic, many studies have been published in the literature in recent years. SCAN, HILBERT, SPIRAL, LMAT, Z-curve, H-curve, and M-curves static path planning models are examined in this study. The localization performances of these path planning models are compared with different performance evaluation criteria using the Weighted Centroid Localization (WCL) technique in the obstacle-presence scenario. The simulation results show the advantages of the H-curve model over existing schemes. The SPIRAL model performs worse than other models in the obstacle-presence scenario.
\end{abstract}

Keywords: localization, mobile anchor node assisted localization, obstacle-handling, path planning, static path planning.

\section{Bazı Statik Yol Planlama Modellerinin Yerelleştirme Performanslarının Engelli Ortamda Karşılaştırılması}

\begin{abstract}
$\ddot{O} \mathbf{z}$
Kablosuz Algılayıcı Ağlar (Wireless Sensor Networks, WSNs)'de bilinmeyen düğümlerin yerelleştirilmesi işlemi için genellikle statik çapalar kullanılmaktadır. Ancak, bunun yerine bütün bilinmeyen düğ̈̈mleri kapsayacak şekilde bir hareketli çapa yörüngesi tasarlamak ve hareketli çapayı bu yörünge boyunca konumunu belirli noktalarda yayınlamak üzere dolaştırmak, daha verimli bir yaklaşım olacaktır. Bu mantıkla, son yıllarda literatürde birçok çalışma yayınlanmıştır. Bu çalışmada, SCAN, HILBERT, SPIRAL, LMAT, Z-eğrisi, Heğrisi ve M- eğrileri statik yol planlama modelleri incelenmiştir. Bu yol planlama modellerinin yerelleştirme performansları, düzgün şekilli engeller içeren ağlarda, Ağırlıklı Merkezi Yerelleştirme (Weighted Centroid Localization, WCL) tekniği kullanılarak farklı performans değerlendirme kriterleriyle karşılaştırılmıştır. Benzetim sonuçları, H-eğrisi modelinin mevcut şemalara göre avantajlarını göstermektedir. SPIRAL modeli ise düzgün şekilli engeller içeren senaryolarda diğer modellere göre daha kötü performans göstermektedir.
\end{abstract}

Anahtar Kelimeler: yerelleştirme, hareketli çapa düğ̈̈m destekli yerelleştirme, engel yönetimi, yol planlama, statik yol planlama.

"Corresponding Author: dogan.yildiz@omu.edu.tr 


\section{Introduction}

Wireless Sensor Networks (WSNs) are the networks that detect application-specific events in the Region of Interest (ROI), collect the processed information about these events wirelessly through multiple sensor nodes and transmit them to a base station. The application areas of WSNs are very diverse. Applications such as environmental monitoring, warfare, child education, surveillance, microsurgery, and agriculture are just a few examples [1]. In most of these applications, the event reported by the sensor nodes becomes meaningful if the event's location is known. Localization is known as the process of determining the physical coordinates of sensor nodes whose locations are unknown [2].

The Global Positioning System (GPS) is one of the most popular positioning technologies easily accessible. However, this technology has some disadvantages, such as high cost and high energy consumption. This situation makes it challenging to install GPS on every node in the WSN. For this reason, GPS units are usually placed in a small number of nodes called Anchor Nodes (ANs) or beacons in the network. The locations of other nodes, except ANs, are obtained by any localization method. These nodes are also called unknown nodes (UNNs) [3].

Many localization methods have been proposed for WSNs until now. These methods can be classified into two general groups according to the distance measurement approaches used in the method: range-based and range-free. Range-based methods use measurement values such as distance or angle to determine the positions of UNNs. They also use extra hardware to obtain these measurements. The most well-known range-based methods are Time of Arrival (ToA), Time Difference of Arrival (TDoA), Angle of Arrival (AoA), and Received Signal Strength Indicator (RSSI). In range-free methods, special equipment is not used for distance estimation. Therefore, they are less costly and simpler than range-based methods. However, range-based methods give more accurate localization results. The most common range-free methods are Approximate Point in Triangle (APIT), Centroid, Distance Vector-hop (DV-hop), and Amorphous [4-7].

The sensor nodes that form WSNs are generally low cost, have low information processing capability, and limited power hardware. There can be hundreds or even thousands of this hardware in a WSN. Therefore, determining the location of the sensors by adding GPS units to each of these hardware is by no means an efficient approach. Therefore, new ideas have to be considered before the sensor nodes in WSN can be localized. With this logic, approaches using only one Mobile Anchor (MA) have been studied in the literature. In these approaches, the only node in the WSN that contains a GPS unit is the MA node and UNNs do not contain a GPS unit. With this idea, significant improvements have been made compared to the scenario where all nodes in the WSN are static. In the Mobile Anchor Node Assisted Localization (MANAL) architecture, MA does not have energy constraints like UNNs. The movement trajectory of the MA can be carefully designed to improve the localization performance of the network. In a localized network using a static anchor, the static anchor cannot be reused after localization. Hence, these nodes will be an extra burden on the network. Fig. 1 shows a MA moving along a certain trajectory by broadcasting periodic signals and the UNNs to be found [8].

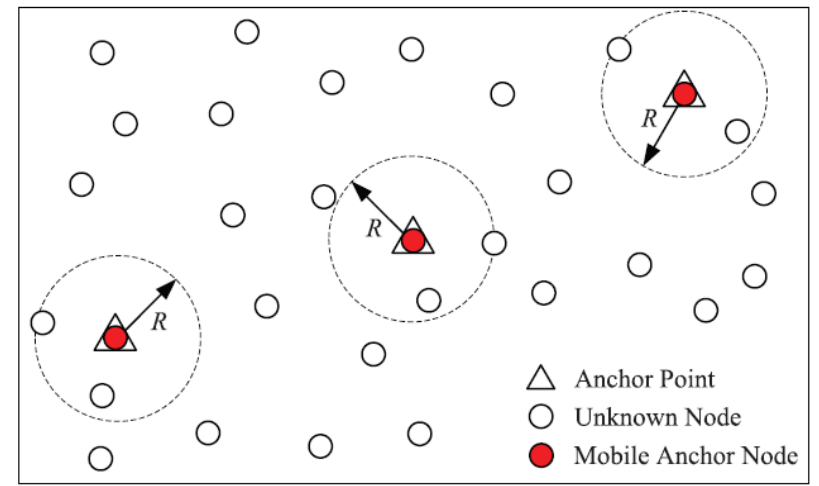

Fig. 1 Mobile anchor node assisted localization [8]

One of the main problems in applications using a MA and unknown static nodes is how to design the path that MA will follow. Generally targeted with such a design are: (i) to find UNNs in the Region of Interest (ROI) with as few errors as possible when the MA uses the path designed; (ii) to find all nodes in the ROI if possible; and (iii) to spend as little energy as possible. In the literature, many studies have been conducted in different scenarios in order to achieve these and similar objectives. In this study, the localization performance of seven static path planning models in the literature is compared in an obstacle-presence environment. The environment used as the base for this comparison is the obstacle environment in the Z-curve study. In addition, a realistic channel is used for the performance evaluation of different mobility models.

\section{Related Work}

As previously mentioned, localization schemes are divided into two classes according to the distance measurement method: range-based and range-free. This classification is very comprehensive. However, given the number of proposed localization schemes and the mobility of known or unknown nodes, expanding this classification has become necessary. Therefore, if this classification is expanded to consider mobility, four main classes are obtained, as shown in Fig. 2 [9].

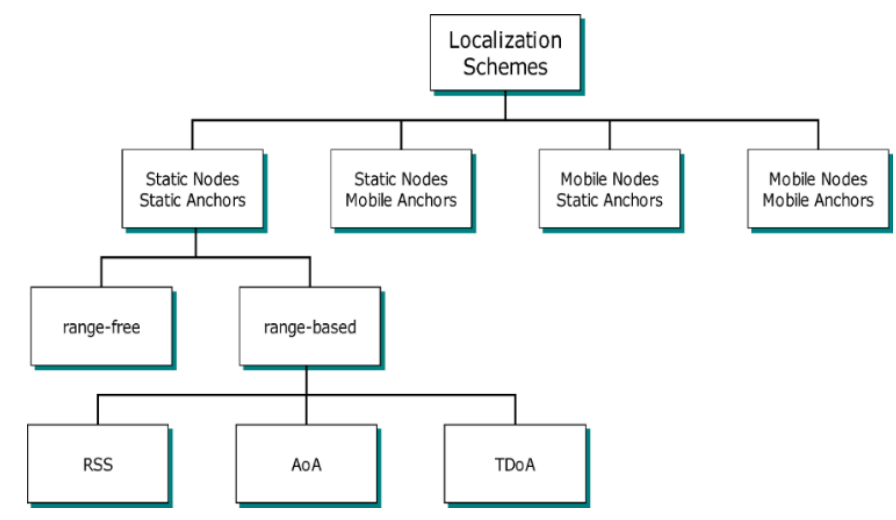

Fig. 2 Classification of localization schemes in WSNs [10]

In this study, studies in the "Static Nodes Mobile Anchor" class, one of the classes in Fig. 2, will be examined. For detailed information about other classes, study [11] can be consulted.

\subsection{Mobile Anchor - Static Nodes}

In this approach, a node with locational information called MA broadcasts this information periodically, allowing the UNNs 
locations to be found according to some localization techniques (e.g., RSSI, TOA, or TDOA). The total cost in the network will be reduced by using a single anchor node. The main problems in MANAL are: i) designing the path of the MA to minimize localization error and performing the localization process of the entire network as soon as possible, ii) designing the localization scheme in which the locations of UNNs are calculated using information from MAs to UNNs [12].

A classification of MA paths is given in Fig. 3. As can be seen, these paths are divided into two main classes as random and planned [9]. Random models are typically used in environments where high localization performances are not required. RandomWay Point (RWP), Random Direction (RD), and Reference Point Group Mobility (RPGM) are examples of these models [13-14].

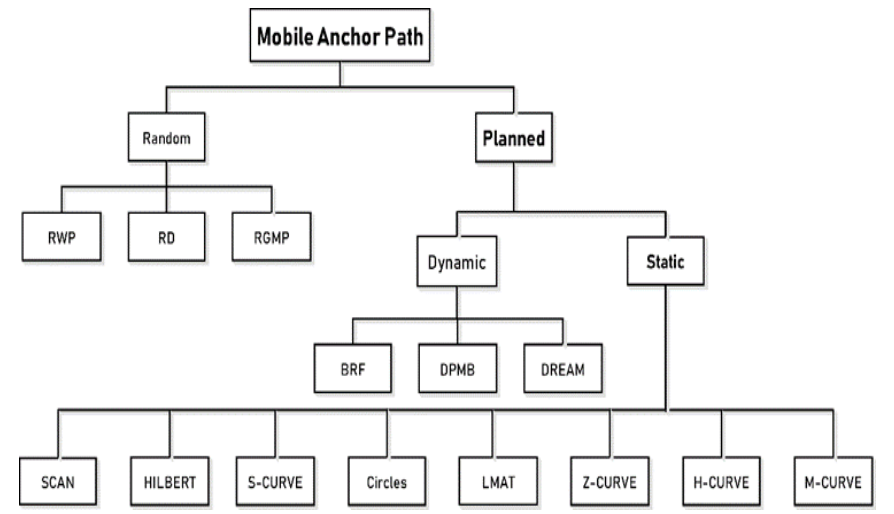

Fig. 3 Different path models for MANAL in WSNs

Planned models can be dynamic or static. In dynamic path planning models, the path is constantly updated with the information obtained on the path according to the need in the application. Therefore, there is no predetermined way.

SCAN, DOUBLE-SCAN, and HILBERT [10], SPIRAL [15], Localization algorithm with a Mobile Anchor node based on Trilateration (LMAT) [16], Z-curve [17], H-curve [9], and Mcurves [18] studies can be given as examples of static path planning models. In static models, the path, starting point, and endpoint to be followed by the MA are determined before the localization process. These were designed before the MA was released to the ROI. Thus, there is no external intervention concerning the trajectory it follows while the MA is in movement. The main problem of these models is the collinearity problem.

In [10], SCAN, DOUBLE-SCAN, and HILBERT path planning models were proposed. These models are known as the first mobile-assisted static path planning models in the literature. In the HILBERT path planning model, the idea of making more turns in the trajectory is proposed to solve the collinearity problem of SCAN and the path length problem of the DOUBLE SCAN. For this purpose, the ROI is divided into four squares of equal size, and the points between the squares are joined. Using different non-collinear position information from MA, UNNs can estimate their position more correctly than the SCAN model. However, one of the most important problems of the HILBERT model is the problem of coverage. Using the HILBERT path planning model, MA cannot provide sufficient information to UNNs located at ROI boundaries to estimate their location. This will increase the localization error and reduce the number of localized nodes. The SCAN and HILBERT path planning models are shown in Fig. 4a and Fig. 4b, respectively.
In [15], the authors proposed a mobile anchor centroid localization (MACL) method. MA is moved on a spiral trajectory. In the localization process, the MA moves and broadcasts the position messages $\left(\mathrm{x}_{\mathrm{i}}, \mathrm{y}_{\mathrm{i}}\right)$ with a spacing $\mathrm{L}$ (moving arc length). The SPIRAL path planning model is shown in Fig. 4c.

Han et al. [16] presented a path planning scheme called LMAT based on trilateration for MAs. In this scheme, the distance between the two locations in which the MA broadcasts location data is defined as the resolution. MA continues its trajectory by forming symmetrical equilateral triangles and broadcasts location data to UNNs at each vertex of these equilateral triangles. This process ensures that the collinearity problem is solved successfully. As a result, high localization accuracy and high localization ratio are achieved with LMAT. However, the length of the path traveled by the MA is long. The LMAT path planning model is shown in Fig. 4d.

Rezazadeh et al. [17] proposed a MA path planning scheme called Z-curve. The MA's path is "Z"-shape. In this study, the authors divided the ROI into squares for three levels and connected the "Z" shapes at each level. As with most mobile path planning models in WSNs, providing three non-collinear points to UNNs in the Z-curve model is one of the main objectives. Additionally, the performance of the Z-curve is evaluated in the presence of obstacles, and the Z-curve obstacle-handling trajectory is proposed to reduce the obstacle problem in localization. The Z-curve model is shown in Fig. 4e.

Alomari et al. [9] presented a MA path planning scheme called H-curves. This scheme is called H-curves because of the multi-curved "H"-shaped paths in the design. The path design was designed to solve the collinearity problem, shorten the distance traveled by MA, and cover each UNN with at least three different MAs. The important point in this study is to create a distance difference of $d_{m} / 2$ between two rows, $d_{m}$ MA's step interval. Thus, the two rows do not overlap, the number of points decreases, and ultimately a triangle-like communication form is created. Weighted Centroid Localization (WCL) and WeightCompensated Weighted Centroid Localization (WCWCL) methods were used as localization estimation methods. The $\mathrm{H}-$ curve path planning model is shown in Fig. $4 \mathrm{f}$.

Kannadasan et al. [18] devised a different path planning scheme that follows MA's trajectory "M" shape. Therefore, they called the proposed scheme "M-curves". In this study, two successive rows are patterned with the letters $\mathrm{M}$ and $\mathrm{W}$. There is a $d_{m}$-long space between $\mathrm{M}$ or $\mathrm{W}$ letters on each row. A $d_{m} / 2$ distance difference is created between two rows. Thus, a trianglelike communication form as in H-curve was obtained, and a solution was made to the collinearity problem. The authors used the "Centroid Method" as the localization estimation method and aimed to increase its performance by adding "Dolphin Swarm Algorithm (DSA)". The M-curve path planning model is shown in Fig. 4g.

The obstacle-handling trajectories of the path planning models in this environment are given in Fig. 4. Except for the Zcurve study, none of the studies in Fig. 4 studied localization performance evaluations in the obstacle-presence environment. This study's main contribution is to measure the performances of the path planning schemes in Fig. 4 in the presence of obstacles and compare them using different evaluation criteria. All path planning models have been tested in the same environment, and this environment is the obstacle-presence environment where a third-order Z-curve is applied [9]. 
European Journal of Science and Technology
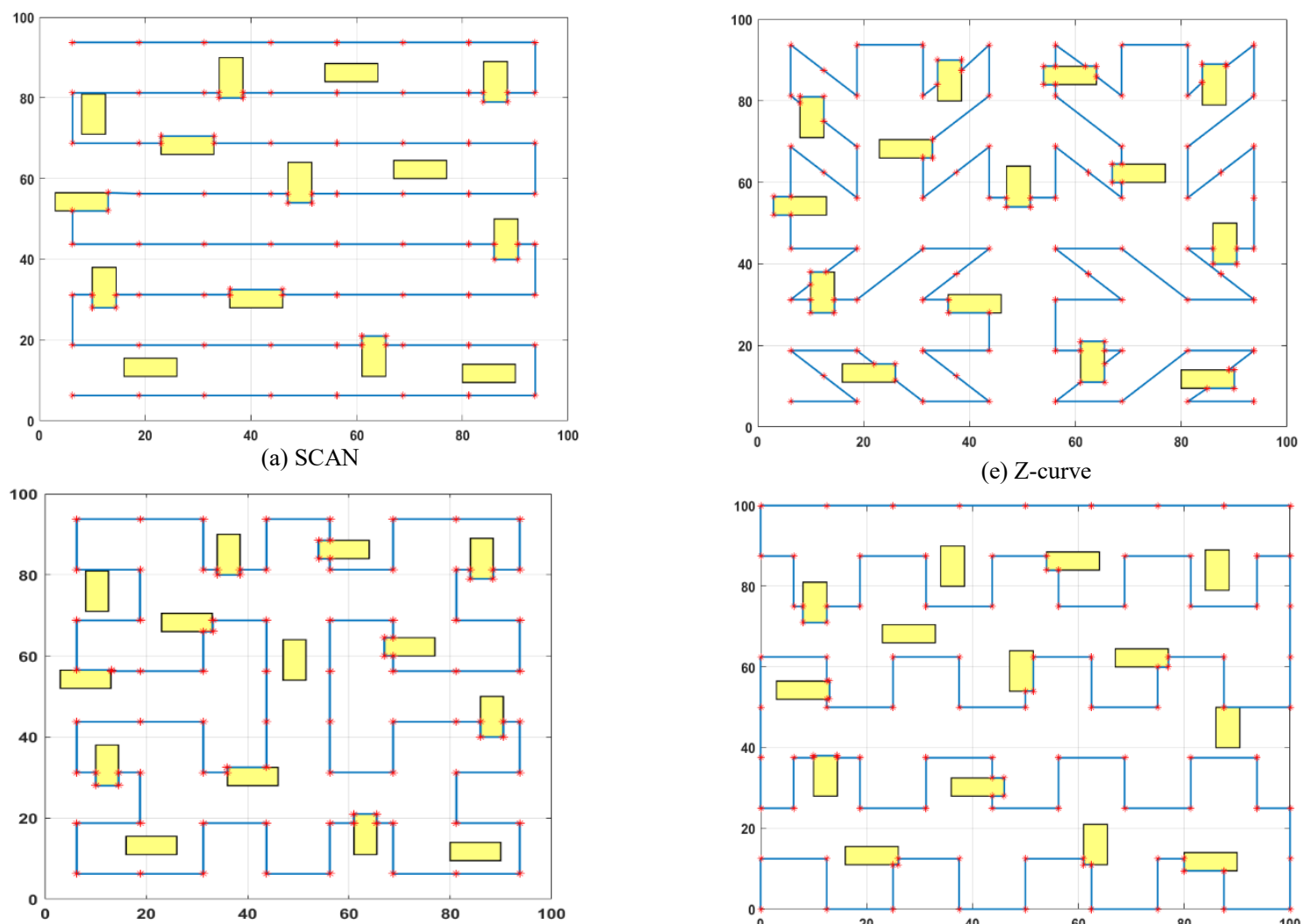

(b) HILBERT

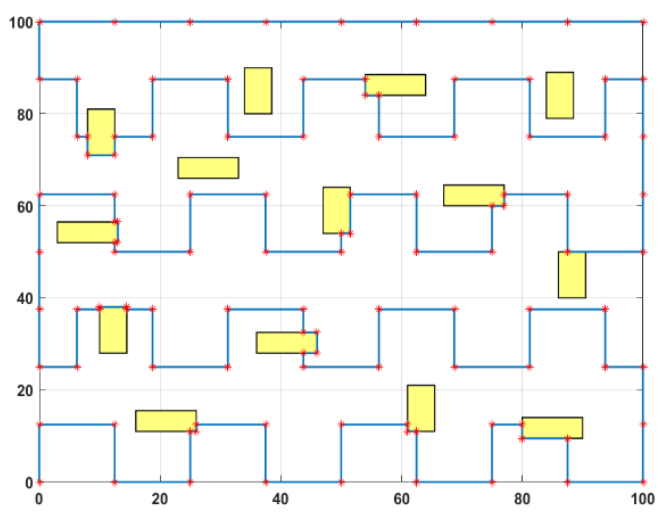

(f) H-curve

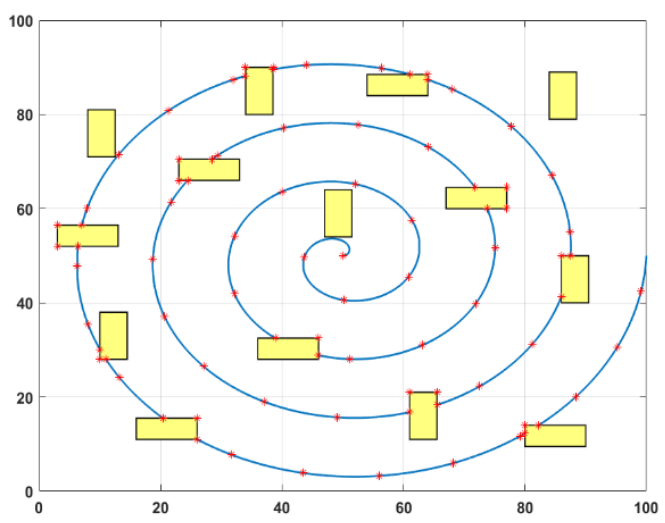

(c) SPIRAL
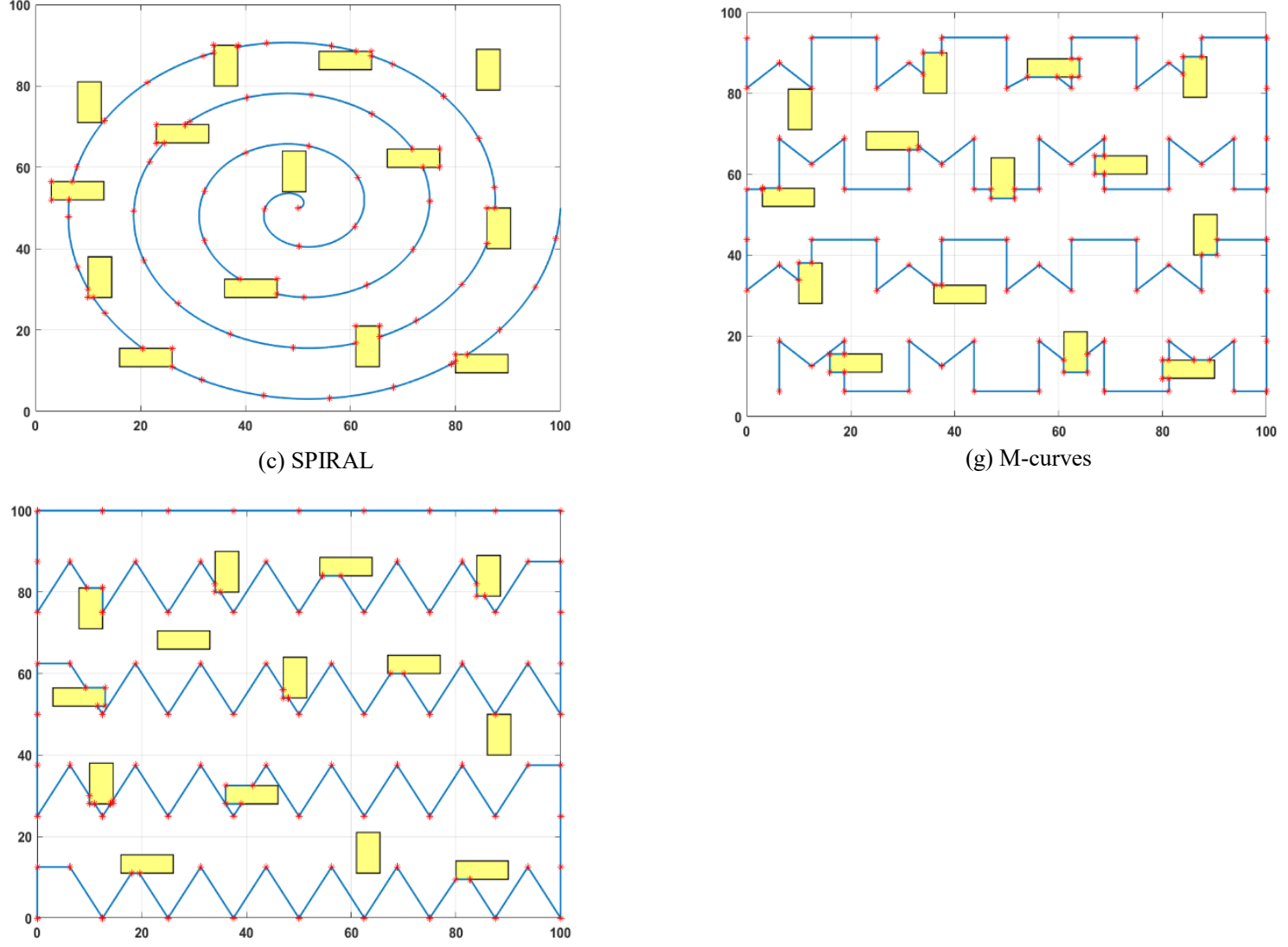

(d) LMAT

Fig. 4 The obstacle-handling trajectories of the path planning models 


\subsection{Localization Scheme}

One of the most fundamental issues in examining the effectiveness of MANAL algorithms is the localization method used to find the locations of UNNs. The efficiency of any path planning model is affected by the type of localization technique. Moreover, since single-hop networks have higher performance than multi-hop networks, scenarios using the single-hop WCL localization technique are considered in this paper.

\subsection{Weighted Centroid Localization (WCL)}

It is a localization method where the locations of UNs are calculated according to the average of the coordinates received from the anchors. Communication consumption and computation cost are low. In the WCL method proposed in the study [19], a $w_{i j}$ weight function was defined based on the RSSI value that varies according to the distance between the UNN and the AN to examine the effect of each $B_{j}(x, y)$ coordinate information received on the location estimation. $w_{i j}$ can be calculated as:

$$
w_{i j}=\frac{1}{\left(d_{i j}\right)^{g}}
$$

where $g$ is a default degree for different situations. Thus, the node location can be formulated as follows ( $n$ indicates the number beacons received from the anchor):

$$
P_{i}=\frac{\sum_{j=1}^{n}\left(w_{i j} \cdot B_{j}(x, y)\right)}{\sum_{j=1}^{n} w_{i j}}
$$

After replacing $w_{i j}$ by $R S S I_{i j}$, the final equation is obtained as:

$$
P_{i}=\frac{\sum_{j=1}^{n}\left(R S S I_{i j} \cdot B_{j}(x, y)\right)}{\sum_{j=1}^{n} R S S I_{i j}}
$$

\section{Network Settings and Assumptions}

The network model used in the path planning model proposed in this study is assumed to have the following features:

1. The UNN and MA are the two types of sensor nodes in the network. N number of UNNs are deployed randomly to the network with uniform distribution.

2. ROI is represented by a two-dimensional area, and a WSN with an $\mathrm{S} \mathrm{m}^{2}$ area size has been established.

3. After the UNNs are distributed to the environment, and they don't know the location information at the first stage. It is assumed that all of these nodes are static, and their positions are unchanged.

4. The communication range (CR) of each sensor node in the network is fixed, and its value is $\mathrm{CR} \mathrm{m}$.

5. An MA has the ability to locate itself at any point in the network, and it can move freely in straight lines, depending on the mobile path planning model across the entire network.

6. The MA stops at certain intervals while moving.

7. The MA sends the location signals from their stopping points to UNNs within the CR. Both the MA and the UNN can communicate with each other only if both are in each other's CRs.

8. The distances from UNNs to MAs are estimated using the RSSI technique.
9. The MA has enough energy to move and also broadcast location information during the localization process. MA consumes more energy than any UNN.

10. The communication model is a channel by log-normal shadowing fading. Taking into account the radio signal propagation loss, we assume that unknown nodes can only receive the beacon packet in a circular region within a communication radius.

11. There is no collision between the MA and the UNNs. That is, MA doesn't go through any UNN.

\subsection{Obstacle-Presence Scenario}

There may be obstacles in the network area in a realistic environment, blocking the MA's path. In the obstacle-resistant trajectory developed in [20] and used in [17], MA detours the obstacle and issues a detour flag at the obstacle's corner points. A UNN uses these flag signals to determine its location. When the MA moves away from the obstacle, it returns to the original trajectory and starts broadcasting standard beacon messages. Since the path planning models discussed in this study are deterministic, the movement pattern and beacon positions for message broadcast are already known. Therefore, the MA can cross the obstacle's edge and continue the path planning pattern where it left off.

In this study, "obstacle-handling trajectory" detailed in [17], briefly mentioned above, is used for all path planning models in the case of obstacle-presence. In this study, this logic is applied to the static path planning models that are examined. In Fig. 5, the obstacle processing trajectory is applied to the H-curve model, one of these models.

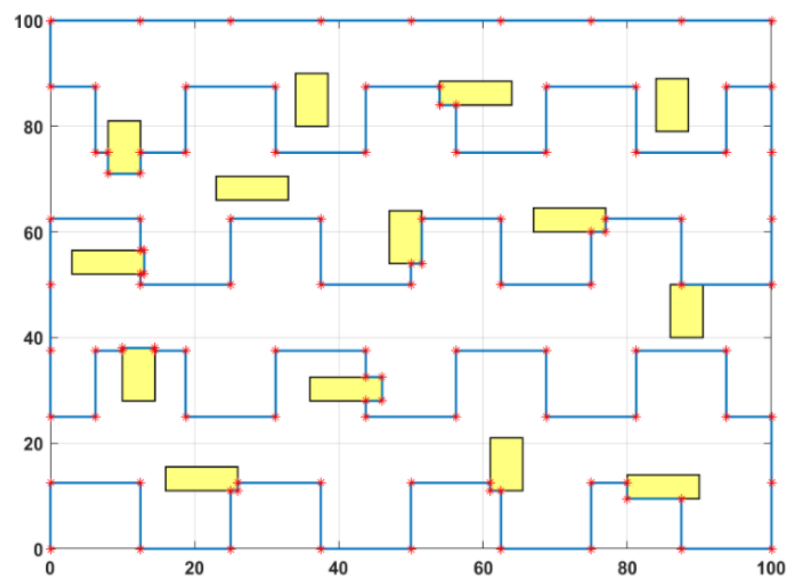

Fig. 5 H-curve in obstacle-handling trajectory

\section{Performance Evaluations}

In this study, the localization performances of seven models in obstacle-presence scenarios were compared. These studies are SCAN, HILBERT, SPIRAL, LMAT, Z-curve, H-curve, and Mcurves studies.

\subsection{Simulation Setup and Wireless Channel}

A realistic wireless model is required to make a reliable assessment. The simulations of the Z-curve [17] take into account the channel model, modulation and coding scheme to extract the relationship between the transmit power and packet reception rate. Chipcon CC1000 radio module was used in the Z-curve study. 
Similarly, the H-curve [9] is another study using realistic parameters. For the wireless model in the H-curve, the characteristics of a wireless node equipped with the Chipcon CC1100 radio module [19] were used. In this study, we also used the wireless channel model and parameters described in [17]. Explanations and specifications for this channel can be found in the following paragraphs. It should also be noted that all path planning models in this study are run under the same realistic channel conditions.

The strength of the signal emitted in the physical environment decreases due to wireless propagation. Therefore, path loss and bit error rate should be modeled for the analysis of the physical layer [21-22]. $P_{r r}$, packet reception ratio means the possibility of a packet being successfully received. It is expressed by the Bernoulli random variable that takes the value 1 if the packet is received and 0 if not. It is given by:

$$
P_{r r}=\left(1-P_{b e}\right)^{8 l}\left(1-P_{b e}\right)^{8(f-l) 2.0}
$$

where $f=20$ byte is the size of the frame related to the TinyOS implementation after being encoded (the frame consists of preamble, network payload and CRC). The Manchester encoding method is used and $P_{b e}$ is the bit error probability which depends on the modulation type. Here, we chose non-coherent FSK modulation which is used in MICA2 motes and formulated by $[21]$ :

$$
P_{b e}=\frac{1}{2} e^{-\frac{S N R B_{N}}{2} R}
$$

where $B_{N}$ is the noise bandwidth and $R$ is the data rate in bits. MICA2 motes use the Chipcon CC1000 radio [23] where $R=$ $19.2 \mathrm{kbps}$ and $B_{N}=30 \mathrm{kHz}$. The signal to noise ratio $(S N R)$ at the receiver is calculated by:

$$
S N R^{d B}=P_{r e c}^{d B}-P_{n}^{d B}
$$

$P_{\text {rec }}$ defines the reception power and $P_{n}$ expresses the noise floor. $P_{n}$ is both environmentally and radio dependent [24], and it is given by:

$$
P_{n}=(F+1) k T_{0} B_{N}
$$

where $F=13 \mathrm{~dB}$ is the noise figure and $k$ is the Boltzmann's constant. $T_{0}=27^{\circ} \mathrm{C}$ is the ambient temperature. In this study, the average noise floor is considered as approximately $-105 \mathrm{dBm}$ $[21,25]$. On the other hand, $P_{r e c}$ is given as:

$$
P_{\text {rec }}{ }^{d B}=P_{\text {trans }}{ }^{d B}-P_{L}^{d B},
$$

where $P_{L}^{d B}$ and $P_{\text {trans }}{ }^{d B}$ are the power loss and transmitting power, respectively. To model the shadowing path loss effect, the log-normal model [24] used:

$$
P_{L}(d)^{d B}=P_{L}\left(d_{0}\right)^{d B}+10 \gamma \log \left(\frac{d}{d_{0}}\right)+X_{\sigma}{ }^{d B},
$$

where $P_{L}(d)^{d B}$ is the power loss after the signal propagates through distance $d, P_{L}\left(d_{0}\right)$ is the power loss at the reference distance $d_{0}, \gamma$ is the path loss exponent and $X_{\sigma}=N\left(0, \sigma^{2}\right)$ is a Gaussian random variable with mean 0 and standard deviation $\sigma$ (shadowing effect).

In this study, the performances of different path planning models are evaluated in MATLAB simulation environment with 50 run times. It is assumed that there are $\mathrm{N}=250$ static UNNs in the ROI, a single MA (M) moving on given path, and the ROI is an area of $\mathrm{S}=100 \times 100 \mathrm{~m}^{2}$. The resolution value $(\mathrm{R})$ is the ratio between the communication range (CR) and the MA step $\left(d_{x}\right)$.
That is, the resolution is expressed in $C R / d_{x}$. Other parameters used in this study are listed in Table 1.

\section{Table 1. Simulation Parameters}

\begin{tabular}{|l|l|l|l|}
\hline Parameters & Symbol & Value & Unit \\
\hline Network size & $\mathrm{S}$ & $100 \times 100$ & $\mathrm{~m}^{2}$ \\
\hline Number of UNNS & $\mathrm{N}$ & 250 & - \\
\hline Number of MAs & $\mathrm{M}$ & 1 & - \\
\hline Resolutions & $\mathrm{R}$ & $1,1.5,2,2.5$ & - \\
\hline Path loss exponent & $\gamma$ & 3.3 & - \\
\hline $\begin{array}{l}\text { Standard deviation of } \\
\text { noise }\end{array}$ & $\sigma$ & $3,5,7,9$ & - \\
\hline Power loss at d & $P_{L}\left(d_{0}\right)$ & 55 & $\mathrm{~dB}$ \\
\hline Reference point & $d_{0}$ & 1 & $\mathrm{~m}$ \\
\hline Transmission power & $P_{\text {trans }}$ & $-20<P_{\text {trans }}<$ & $\mathrm{dBm}$ \\
\hline Simulation run & - & 50 & \\
\hline
\end{tabular}

\subsection{Accuracy}

One of the most important criteria for evaluating any proposed model is the accuracy of localization. Therefore, this criterion is considered as the primary criterion used to compare different path planning models. Accuracy is related to localization error by the number of nodes. In this study, two methods are used to calculate the localization error of each model: the average localization error and the standard deviation of the localization error.

\subsubsection{Average Localization Error}

The average localization error is used to assess how accurate the estimated position obtained is. The ratio of the sum of the localization errors of all the localized UNNs in the ROI to the number of nodes determines the average error rate. The localization error for node $\mathrm{i}$ is calculated as follows:

$$
\operatorname{error}_{(i)}=\sqrt{\left(x_{i}-u_{i}\right)^{2}+\left(y_{i}-v_{i}\right)^{2}}
$$

$\left(x_{i}, y_{i}\right)$ are the actual coordinates of the node discussed and $\left(u_{i}, v_{i}\right)$ are the estimated coordinates of the same node. Therefore, error $_{\text {avg }}$ is given by the average localization error equation for the total $N$ sensor nodes.

$$
\text { error }_{\text {avg }}=\left(\sum_{i=1}^{N} \operatorname{error}_{(i)}\right) / N
$$

As shown in Fig. 6, in most places, the H-curve path planning model showed the best error performance compared to other models. After this model, the best error result is obtained with the LMAT model. Although the error performance of other models is similar, the SPIRAL model is noticeably worse than other models. If we pay attention to the path trajectories of the H-curve and LMAT models in Fig. 4, it is observed that the MA passes through the whole ROI. In addition, beacons are broadcasted at the borders. This is why these models localize UNNs with less error. With the SPIRAL model, it is not possible to send messages to the entire ROI. Thus, a higher error rate is achieved. 


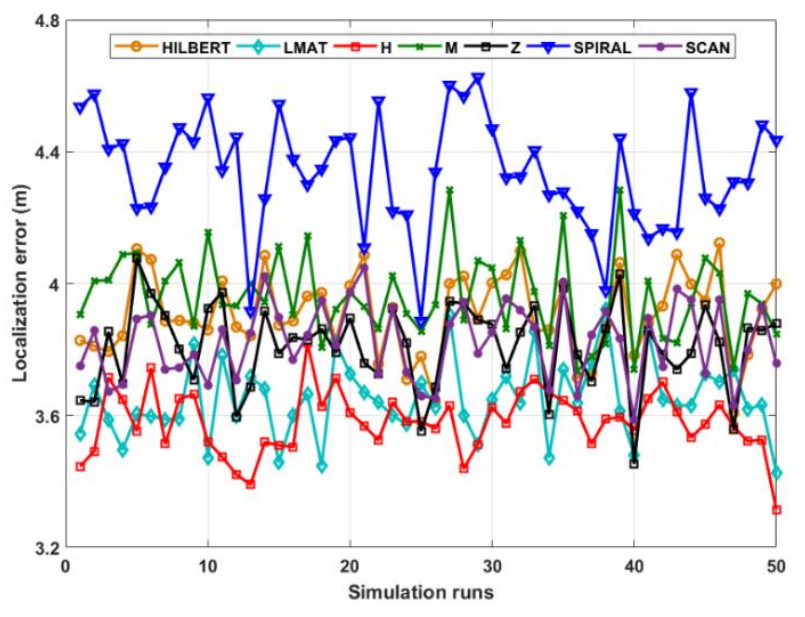

Fig. 6 Localization errors of all mobile path planning models

In this study, the average localization error variation with two variables given in Table 1 is also investigated separately: resolution $(\mathrm{R})$ and standard deviation (std) of noise $(\sigma)$. Firstly, $250 \mathrm{UNNs}$ simulations with different resolutions ranging from 1 to 2.5 are performed. Fig. 7 shows the average localization error according to the resolution values for path models.

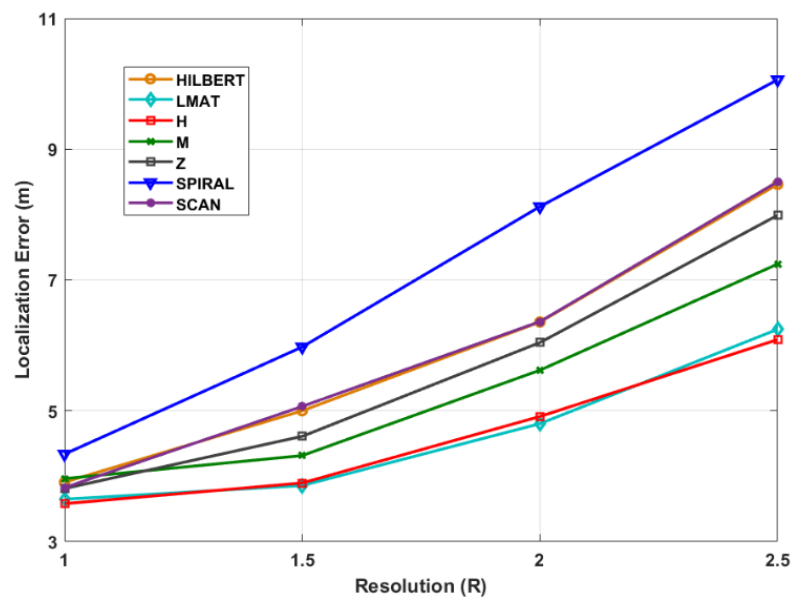

Fig. 7 Average localization errors of models by resolution

Fig. 7 shows a graph showing how the localization error varies according to the resolution. As seen from the figure, the $\mathrm{H}$ curve and LMAT path planning models are superior to other models. The SPIRAL model, on the other hand, showed poor performance compared to other models due to its path design. Also, it can be said that as the resolution value increases for all models, the localization error also increases.

Secondly, in Fig. 8, the same parameters are repeated with different $\sigma$ values for fixed resolution. Fig. 8 shows the average localization error for path planning models with different $\sigma$ values when the WCL algorithm is applied. It is the model H-curve with the smallest localization error for all $\sigma$ values. If the figure is examined carefully, it can be said that the error performance of most models changes little with changes in $\sigma$. The reason that the localization error does not vary much with $\sigma$ is the characteristic of the WCL method that takes the weighted average of the beacon locations.

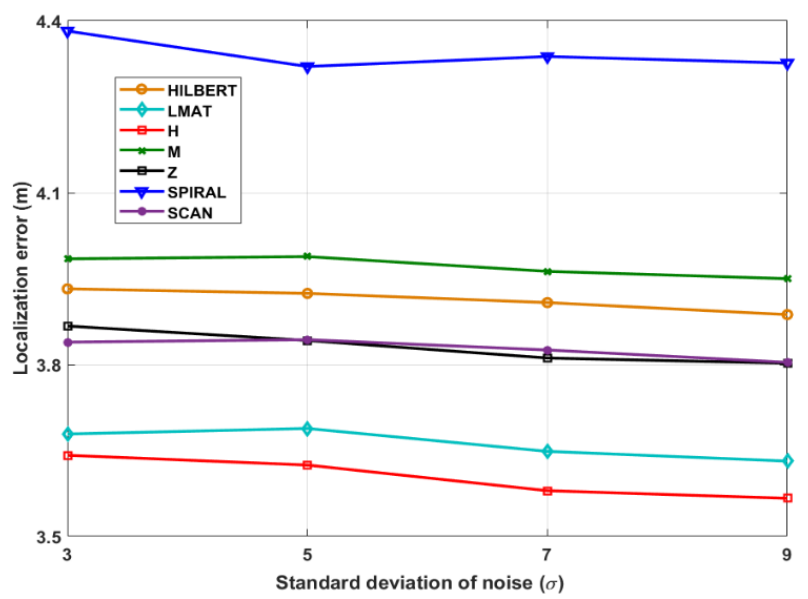

Fig. 8 Average localization errors of the models according to the std of the noise $(\mathrm{R}=1)$

\subsubsection{Standard Deviation of the Localization Error}

The low standard deviation of error values indicates that most of the values are close to the mean. The standard deviation of the localization error is:

$$
\operatorname{error}_{s t d}=\sqrt{\frac{1}{N} \sum_{i=1}^{N}\left(\operatorname{error}_{(i)}-\operatorname{error}_{(a v g)}\right)^{2}}
$$

where $N$ is the number of UNNs, error $_{(i)}$ is the localization error for node $i$, and error $_{(a v g)}$ is the average localization error.

Fig. 9 shows the std of the localization error for each path planning model for WCL. For each simulation, $\mathrm{R}=1$ and $\sigma=7$. As shown in Fig. 9, the std of the error performance of the $\mathrm{H}$ curve path planning model for the WCL method is better than the other models. The LMAT model is similar to this model. The SPIRAL model is again the worst performing method in this scenario. This means that the errors obtained by the SPIRAL method are mostly far from the average.

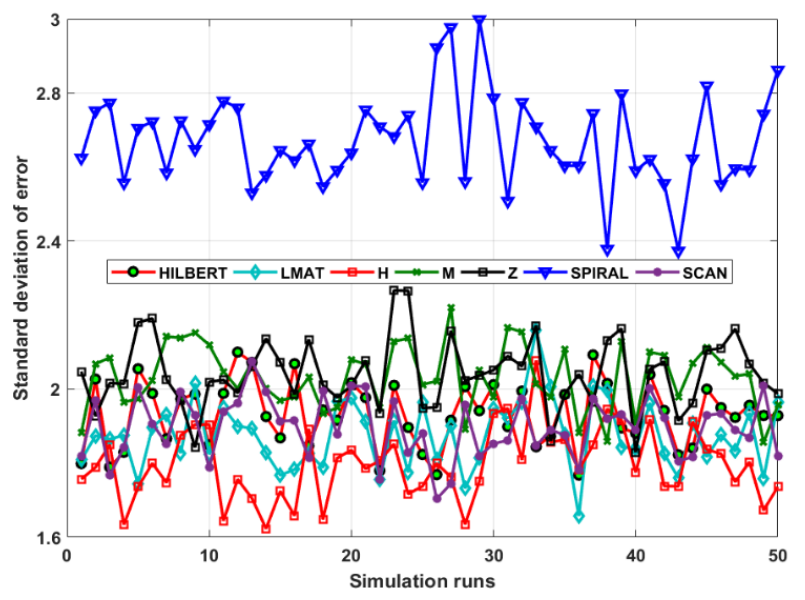

Fig. 9 Std of errors of all path planning models

The average of the 50 simulations is then calculated using different $\mathrm{R}$ values, as shown in Fig. 10. In Fig. 10, $\sigma=7$ and $\mathrm{R}$ vary between 1 and 2.5. This figure shows the std of the localization error for different resolution values. When the performance of the models for the WCL method is evaluated, it can be said that the error performance of the models is slightly different from the average. As the resolution increases, the std of the models' error value increases compared to the previous 
resolution. LMAT and H-curve models show the best std of error performance. According to the same evaluation criteria, the SPIRAL model again shows the worst result. Also, it is interesting to note that the SCAN and HILBERT models proposed in the same study give roughly the same performance at all resolutions according to this evaluation criterion.

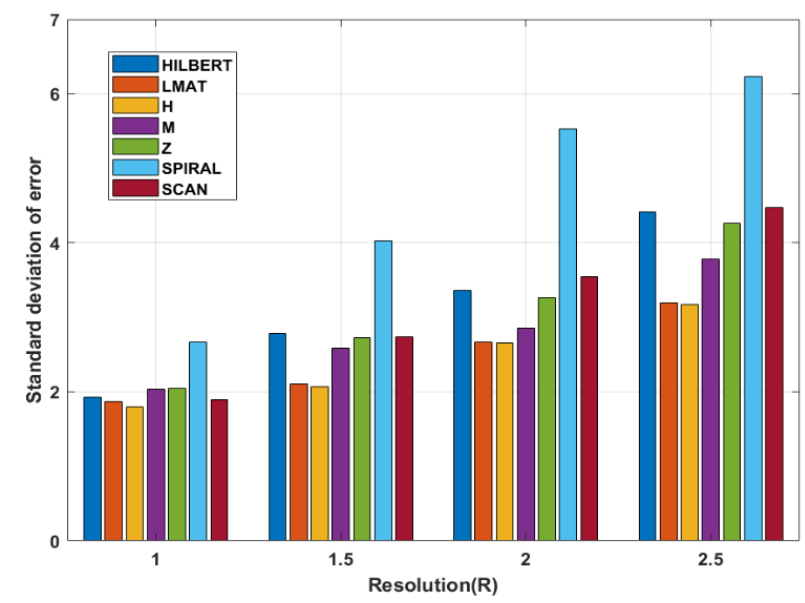

Fig. 10 Std of errors of the path planning models according to resolution $(\sigma=7)$

\subsection{Localization Ratio}

Coverage or localization ratio indicates the number of nodes successfully localized by the number of UNN. As many localized nodes as possible are requested for each model implemented at the end of the localization process. In the scenario created in Fig. 11 , localization ratio performances of models with different resolutions and constant $\sigma=7$ for 250 UNNs are examined. If the localization ratio performances of the models are evaluated, it can be said that all models show high performance in general. However, the SPIRAL method demonstrated significantly lower localization ratio performance than other methods for $\mathrm{R}<2$. Generally, the reason for the high localization rate performance is that the localization technique used is WCL.

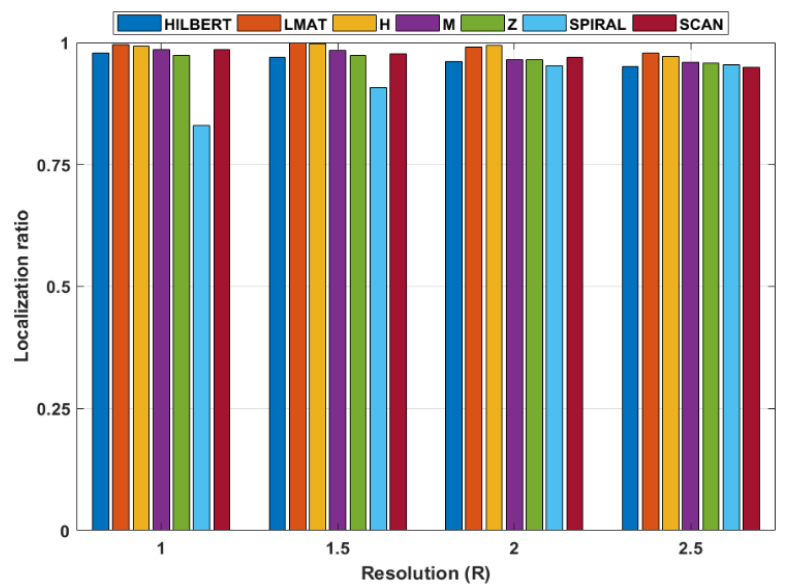

Fig. 11 Localization ratio of the path planning models by resolution $(\sigma=7)$

\subsection{Path Length}

The path length is the length of the distance MA travels, following the proposed model as it moves through the network. Path length does not affect on the localization error, but it helps find parameters such as time and energy spent for the entire localization process. Mathematical equations of path length designs are calculated according to two variables: $S$ network size and $d x$ the distance between both points. The following equations show the mathematical expressions of each static path planning model. Equations (16), (17), (19), (20), (21), (22), and (23) show the path length of SCAN, HILBERT, SPIRAL, LMAT, Z-curve, $\mathrm{H}$-curve, and M-curves path planning models, respectively.

For example, if $d x=12.5 \mathrm{~m}, S=64(d x)^{2}$ is obtained. However, as can be shown from Figure 4 and Figure 5, $d x$ is not always $12.5 \mathrm{~m}$ in all models.

$$
\begin{gathered}
L_{S C A N}=\frac{s}{d x}-d x \\
L_{\text {Hilbert }}=\frac{s}{d x}
\end{gathered}
$$

The trajectory of SPIRAL is calculated using the following equation:

The trajectory length of SPIRAL is calculated by:

$$
\begin{gathered}
x=x_{0}+r \times t \times \cos (2 \pi t) \\
y=y_{0}+r \times t \times \sin (2 \pi t) \\
L_{S P I R A L}=\sum_{t=1}^{\left[\frac{\sqrt{S}}{r}\right]} \sqrt{r^{2}+4 r^{2} \pi^{2} t^{2}+4 r^{2} t \sin 4 \pi t} \\
L_{L M A T}=\frac{s}{d x}+20 d x \\
L_{Z}=\left[\left(\frac{5}{8} \times 4^{3}\right)-1\right] d x+\left[\left(\frac{3}{8} \times 4^{3}\right)\right] \times \sqrt{2} d x \\
L_{H}=\frac{s}{d x}+18 d x \\
L_{M}=\frac{109}{2} d x+16 \sqrt{2} d x
\end{gathered}
$$

Fig. 12 shows the path lengths of the path planning models for the obstacle-presence scenario. The SPIRAL model is the model that travels the shortest distance with $652.21 \mathrm{~m}$. HILBERT takes a little longer with a travel distance of approximately 833 $\mathrm{m}$. But, these models suffer from the coverage problem. The LMAT and H-curve travel longer distances than other models. However, according to most evaluation criteria, these models give better results than other models. These results show that when path length and other performance criteria are evaluated together, H-curve and LMAT static path planning models are the optimum models that can be used in obstacle-presence scenarios.

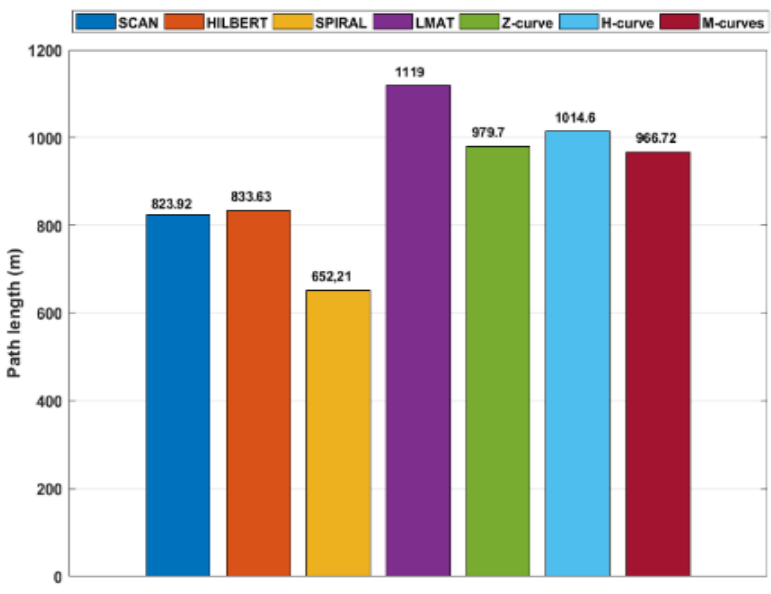

Fig. 12 The path length for the different mobility models in obstacle-presence scenario 


\section{Conclusions and Future Work}

In this study, the localization performances of SCAN, HILBERT, SPIRAL, LMAT, Z-curve, H-curve, and M-curve path planning models are compared with different performance evaluation criteria using the WCL technique in the obstaclepresence scenario. According to these performance criteria, the $\mathrm{H}$ curve model, followed by the LMAT model, showed the best performance. On the other hand, the worst performance was generally achieved with SPIRAL path planning. The reason for poor performance in this model is that the broadcast signals cannot cover the ROI due to the MA trajectory. The same problem exists in SCAN and HILBERT. follows:

Our future research topics in MANAL will likely be as

i. Another of our future studies will be to compare the energy consumption and energy efficiency of static path planning models in the literature.

ii. Observing the performance of the models in irregular obstacle-presence areas. To design a suitable dynamic path planning model for such areas.

iii. Using only one anchor, MANAL algorithms can take a long time to find all UNNs in an ROI, especially for large-scale WSNs. Therefore, the collaborative MANAL algorithm using several MAs can be designed to reduce localization time and improve localization accuracy.

\section{References}

[1] Puccinelli, D., \& Haenggi, M. (2005). Wireless sensor networks: applications and challenges of ubiquitous sensing. IEEE Circuits and systems magazine, 5(3), 19-31.

[2] Mao, G., Fidan, B., \& Anderson, B. D. (2007). Wireless sensor network localization techniques. Computer networks, 51(10), 2529-2553.

[3] Cheng, L., Wu, C., Zhang, Y., Wu, H., Li, M., \& Maple, C. (2012). A survey of localization in wireless sensor network. International Journal of Distributed Sensor Networks, 8(12), 962523.

[4] Singh, S. P., \& Sharma, S. C. (2015). Range free localization techniques in wireless sensor networks: A review. Procedia Computer Science, 57, 7-16.

[5] Yildiz, D., Karagol, S., \& Ozgonenel, O. (2017, April). A hyperbolic location algorithm for various distributions of a Wireless Sensor Network. In 2017 5th International Istanbul Smart Grid and Cities Congress and Fair (ICSG) (pp. 75-79). IEEE.

[6] Mondal, K., Karmakar, A., \& Mandal, P. S. (2016). Path planning algorithms for mobile anchors towards range-free localization. Journal of Parallel and Distributed Computing, 97, 35-46.

[7] He, T., Huang, C., Blum, B. M., Stankovic, J. A., \& Abdelzaher, T. (2003, September). Range-free localization schemes for large scale sensor networks. In Proceedings of the 9th annual international conference on Mobile computing and networking (pp. 81-95).

[8] Han, G., Jiang, J., Zhang, C., Duong, T. Q., Guizani, M., \& Karagiannidis, G. K. (2016). A survey on mobile anchor node assisted localization in wireless sensor networks. IEEE Communications Surveys \& Tutorials, 18(3), 2220-2243.
[9] Alomari, A., Comeau, F., Phillips, W., \& Aslam, N. (2018). New path planning model for mobile anchor-assisted localization in wireless sensor networks. Wireless Networks, 24(7), 2589-2607.

[10] Koutsonikolas, D., Das, S. M., \& Hu, Y. C. (2007). Path planning of mobile landmarks for localization in wireless sensor networks. Computer Communications, 30(13), 25772592.

[11] Han, G., Xu, H., Duong, T. Q., Jiang, J., \& Hara, T. (2013). Localization algorithms of wireless sensor networks: a survey. Telecommunication Systems, 52(4), 2419-2436.

[12] Han, G., Zhang, C., Lloret, J., Shu, L., \& Rodrigues, J. J. (2014). A mobile anchor assisted localization algorithm based on regular hexagon in wireless sensor networks. The Scientific World Journal, 2014.

[13] Johnson, D. B., \& Maltz, D. A. (1996). Dynamic source routing in ad hoc wireless networks. In Mobile computing (pp. 153-181). Springer, Boston, MA.

[14] Han, G., Chao, J., Zhang, C., Shu, L., \& Li, Q. (2014). The impacts of mobility models on DV-hop based localization in mobile wireless sensor networks. Journal of Network and Computer Applications, 42, 70-79.

[15] Hu, Z., Gu, D., Song, Z., \& Li, H. (2008, July). Localization in wireless sensor networks using a mobile anchor node. In 2008 IEEE/ASME international conference on advanced intelligent mechatronics (pp. 602-607). IEEE.

[16] Han, G., Xu, H., Jiang, J., Shu, L., Hara, T., \& Nishio, S. (2013). Path planning using a mobile anchor node based on trilateration in wireless sensor networks. Wireless Communications and Mobile Computing, 13(14), 1324-1336.

[17] Rezazadeh, J., Moradi, M., Ismail, A. S., \& Dutkiewicz, E. (2014). Superior path planning mechanism for mobile beacon-assisted localization in wireless sensor networks. IEEE Sensors Journal, 14(9), 3052-3064.

[18] Kannadasan, K., Edla, D. R., Kongara, M. C., \& Kuppili, V. (2019). M-curves path planning model for mobile anchor node and localization of sensor nodes using dolphin swarm algorithm. Wireless Networks, 1-15.

[19] Blumenthal, J., Grossmann, R., Golatowski, F., \& Timmermann, D. (2007, October). Weighted centroid localization in zigbee-based sensor networks. In 2007 IEEE international symposium on intelligent signal processing (pp. 1-6). IEEE.

[20] Ou, C. H., \& He, W. L. (2012). Path planning algorithm for mobile anchor-based localization in wireless sensor networks. IEEE Sensors Journal, 13(2), 466-475.

[21] Zamalloa, M. Z., \& Krishnamachari, B. (2007). An analysis of unreliability and asymmetry in low-power wireless links. ACM Transactions on Sensor Networks (TOSN), 3(2), 7-es.

[22] Dezfouli, B., Radi, M., Abd Razak, S., Hwee-Pink, T., \& Bakar, K. A. (2015). Modeling low-power wireless communications. Journal of Network and Computer Applications, 51, 102-126.

[23] Chipcon, Dallas, TX, USA. CC1000 Low Power Radio Transceiver [Online]. Available: https://www.ti.com/product/CC1000 Accessed 11 June 2020.

[24] Rappaport, T. S. (1996). Wireless communications: principles and practice (Vol. 2). New Jersey: prentice hall PTR.

[25] Srinivasan, K., Dutta, P., Tavakoli, A., \& Levis, P. (2010). An empirical study of low-power wireless. ACM Transactions on Sensor Networks (TOSN), 6(2), 1-49. 